\title{
25 Research Suare \\ Novel RIPK1 Mutations Causing Infantile-onset IBD with Inflammatory and Fistulizing Features
}

\author{
Mutaz Sultan \\ Mohammad Adawi \\ Nitzan Kol \\ Blake McCourt \\ Ihda Adawi \\ Lael Werner \\ Atar Lev \\ Scott Snapper \\ Ortal Barel
}

Liza Konnikova

Raz Somech

Dror S. Shouval ( $\sim$ dror.shouval@gmail.com )

Edmond and Lily Safra Children's Hospital https://orcid.org/0000-0001-5980-2954

\section{Research Article}

Keywords: RIPK1, monogenic IBD, VEOIBD, necroptysis, inflammasome, Crohn's

Posted Date: February 24th, 2022

DOI: https://doi.org/10.21203/rs.3.rs-1347173/v1

License: (c) (1) This work is licensed under a Creative Commons Attribution 4.0 International License.

Read Full License 


\section{Abstract}

Purpose: Receptor-interacting serine/threonine-protein kinase 1 (RIPK1) is an important regulator of necroptosis and inflammatory responses. We present the clinical features, genetic analysis and immune work-up of two patients with infantile-onset inflammatory bowel disease (IBD) resulting from novel RIPK1 mutations.

Methods: Whole exome and Sanger sequencing was performed in two IBD patients. Mass cytometry time of flight (CyTOF) was conducted for in-depth immunophenotyping on one of the patient's peripheral blood mononuclear cells, and compared to control subjects and patients with Crohn's disease.

Results: The patients presented with severe colitis and perianal fistulas in the first months of life, without severe/atypical infections. Genetic studies identified novel and pathogenic genetic variants in RIPK1. Using CyTOF unbiased clustering analysis, we identified peripheral immune dysregulation in one of these patients, characterized by an increase in IFNy $\mathrm{CD}^{+} \mathrm{T}$ cells along with a decrease in monocytes, dendritic cells and B cells. Moreover, RIPK1-deficient patient's immune cells exhibited decreased IL-6 production in response to LPS across multiple cell types including T cells, B cells and innate immune cells.

Summary: Mutations in RIPK1 should be considered in very young patients presenting with colitis and perianal fistulas. Given RIPK1's role in inflammasome activation, but also in epithelial cells, it is unclear whether IL1 blockade or allogeneic hematopoietic stem cell transplantation can suppress or cure the hyper-inflammatory response in these patients. Additional studies in humans are required to better define the role of RIPK1 in regulating intestinal immune responses, and how treatment can be optimized for patients with RIPK1 deficiency.

\section{Introduction}

Significant progress has been made in the last 15 years in understanding the role of genetics in the pathogenesis of inflammatory bowel disease (IBD), including Crohn's disease (CD) and ulcerative colitis (UC) ${ }^{1}$. More than 240 single nucleotide polymorphisms, typically with a relatively high mean allelic frequency (MAF) in the general population, have been identified as conferring risk for $C D, U C$ or both ${ }^{2}$. On the other hand, rare deleterious variants directly causing intestinal inflammation were identified using advanced sequencing technologies ${ }^{3,4}$. To date, nearly a 100 distinct monogenic disorders associated with IBD were characterized, resulting from deleterious and rare mutations in genes important for immune responses, epithelial cell function, or both ${ }^{5,6}$. Consequently, in many of these diseases intestinal inflammation is accompanied by an immunodeficiency state, manifesting as recurrent or atypical infections. Identification of a monogenic disorder can have a marked impact on care provided for these patients through the possibility of providing targeted therapies, as we and others have shown before with anakinra for patients with IL 10 receptor mutations ${ }^{7}$ and abatacept for LPS Responsive Beige-Like Anchor Protein (LRBA) deficiency ${ }^{8}$. 
Mutations in Receptor-interacting serine/threonine-protein kinase 1 (RIPK1) associated with monogenic IBD were recently reported ${ }^{9,10}$. The RIPK1 pathway along with other kinases, including RIPK3 and mixedlineage kinase domain-like pseudokinase $(M L K L)^{11}$ is important for necroptosis, a regulated necrotic cell death mechanism. We present the clinical course, genetic analysis and immune work-up of two patients from unrelated families who presented with severe infantile-onset IBD and were found to harbor novel deleterious mutations in RIPK1.

\section{Methods}

\section{Whole exome sequencing}

The study was approved by the IRB committee at Sheba Medical Center and informed consent was obtained from all individual participants included in the study or their parents. Whole exome sequencing (WES) was performed using an Agilent v5 Sureselect capture kit and Illumina 2500 sequencing technology. Paired end reads (2X100 bp) were obtained, processed and mapped to the genome. The average sequencing depth of the target region is $92 X$ with $95.63 \%$ of bases reached at least 10X coverage. We used the BWA mem algorithm (version 0.7.12) ${ }^{12}$ for alignment of the sequence reads to the human reference genome (hg19). The HaplotypeCaller algorithm of GATK version 3.4 was applied for variant calling, as recommended in the best practice pipeline ${ }^{13}$. KGG-seq v.08 was used for annotation of identified variants ${ }^{14}$ and in house scripts were applied for filtering based on family pedigree and local dataset of variants detected in previous sequencing projects. Likely pathogenicity was assessed if the variant was truncating (splicing or non-sense) or missense; in-frame indels were considered if they were predicted to be pathogenic by online prediction tools including PolyPhen-2, SIFT, CADD and MutationAssessor.

\section{Immune work-up}

Cell surface markers of peripheral blood mononuclear cells (PBMCs) were determined by immunofluorescent staining using flow cytometry (Navios, Beckman Coulter, Brea, CA, US) with antibodies purchased from Beckman Coulter. T cell receptor excision circles (TREC) analysis was performed using DNA extracted from the patient's PBMCs. The amount of signal joint TREC copies per DNA content was determined by real-time quantitative PCR as previously described ${ }^{15}$.

\section{Sanger sequencing}

Exons 4 and 11 of RIPK1 gene were amplified and sequenced by the Sanger method. Briefly, polymerase chain reaction (PCR) amplification was performed using three sets of the following primers:

exon 4-Fw: CAGAATTTCATGTGAACGTTTCCT

exon 4 -Rw: GGCTAAGTCCTCACAAGCAGAA 
exon 11 Rw: CCCATTCTCCAGCTATGAAGTACA

The PCR reaction took place in a $25-\mu \mathrm{L}$ volume containing $50 \mathrm{ng}$ of DNA, $10 \mathrm{ng}$ of each primer, $1.5 \mathrm{mM}$ dNTPs, in $1.5 \mathrm{mM} \mathrm{MgCl}$, PCR buffer, with 1.2 units of Taq polymerase (Bio-Line, London, UK). After an initial denaturation of $5 \mathrm{~min}$ at $95^{\circ} \mathrm{C}, 30$ cycles were performed $\left(94^{\circ} \mathrm{C}\right.$ for $30 \mathrm{~s}, 60^{\circ} \mathrm{C}$ for $30 \mathrm{~s}$, and $72^{\circ} \mathrm{C}$ for $30 \mathrm{~s}$ ), followed by a final extension of $10 \mathrm{~min}$ at $72^{\circ} \mathrm{C}$. PCR amplicons were sequenced in both directions using a commercial sequencing service [Hy Laboratories, Ltd. (Hylabs) Park Tamar Rehovot, Israel].

\section{Mass cytometry (CyTOF) studies}

Peripheral blood mononuclear cells from the a RIPK1-deficient patient, both parents, three healthy controls and three patients with active CD were stained with a panel of metal-chylated surface antibodies targeting markers of major immune cell lineages (Supplementary Table 1) per previously published protocol $^{16}$. The samples were run on Helios2 mass cytometer (Fluidigm, San Francisco, CA, USA). FCS files obtained were analyzed with premium Cytobank software and pre-gated on CD $45^{+} / \mathrm{viable} /$ single/DNA ${ }^{+}$events before initiating the analysis. Normalization beads were used and gated out of the analysis. The data was automatically clustered with Pheongraph through cytofkit package in R and visualized with tSNE using all leukocytes (CD45 ${ }^{+}$cells) as input and manually labeled based on markers expressed in the individual clusters. Cluster abundance (\% of CD $45^{+}$viable single events) and fold change over the RIPK1 abundance were computed and plotted for comparison between groups using Prism8 software. Additionally, clusters of similar cellular subtype were combined, and similarly, the ratio of CD4/CD8 cells was calculated.

To identify dysregulation in activation and cytokine production of the immune cells in RIPK1 deficiency, we stimulated PBMCs from Patient 1 and his parents with either lipopolysaccharide (LPS) or phorbol 12myristate 13-acetate (PMA) and lonomycin. PBMC's from patient and parents were thawed and washed with $T$ cell media, then centrifuged at $300 \mathrm{~g}$. The supernatant was discarded and pelleted PBMC's were resuspended in $1 \mathrm{ml}$ of T Cell media. GolgiStop and GolgiPlug were then added to each stimulation tube and each sample was stimulated with either LPS $1 \mathrm{mg} / \mathrm{ml}$ or PMA $0.5 \mathrm{ng} / \mathrm{ml}$ and lonomycin $1 \mathrm{mg} / \mathrm{ml}$ for 4 hours at $37^{\circ} \mathrm{C}$. Samples were then centrifuged and washed again in T cell media then prepped for CyTOF where they were stained with heavy metal chelated antibodies (Supplementary Table 2) first for surface antigens, then fixed and permeabilized and finally stained with antibodies for intracellular antigens ${ }^{16}$. All samples were analyzed as above. In addition, mean expression of various cytokines (mean metal intensity) was calculated in the various populations indicated.

\section{Results}

\section{Case description}


Patient 1 was referred at the age of 16 months for evaluation of IBD. He was born to a consanguineous Muslim family (parents first degree cousins) and presented at the age of 9 months with fever and diarrhea that were initially attributed to amebiasis. Despite metronidazole treatment his condition deteriorated, and he developed perianal abscesses with multiple fistulas (Figure 1A). Blood tests were significant for anemia (hemoglobin 6.5 gram/dL), hypoalbuminemia (albumin $1.5 \mathrm{gr} / \mathrm{dL}$ ) and elevated inflammatory markers (CRP $94 \mathrm{mg} / \mathrm{L}$, normal $<5 \mathrm{mg} / \mathrm{L}$ ). Due to the severity of the perianal disease, the patient underwent double barrel protective ileostomy. Despite this intervention, his condition did not improve. At the age of 16 months the patient appearead cachectic. Weight was $7.4 \mathrm{~kg}$ (Z score -4), length $73 \mathrm{~cm}$ (Z score -2) and head circumference $46 \mathrm{~cm}$ (Z score -2). Abdominal exam showed double barrel ileostomy, with severe inflammation in the surrounding skin, and multiple perianal fistulas with deep ulcerations and perianal fissures. Colonoscopy demonstrated patchy areas of

severe colonic ulcerations with pseudopolyps, with normal mucosa in between, while an upper endoscopy was unremarkable. Colonic biopsies revealed chronic active inflammation with focal cryptitis (Figure 1B).

Following a course of broad-spectrum antibiotics and nutritional supplementation, the patient was started on metronidazole, mesalamine and azathioprine, and later began adalimumab. Nevertheless, the patient failed to respond to this TNFa antagonist despite adequate drug levels and absence of anti-drug antibodies, thus experiencing primary pharmacodynamic failure, and at the age of 2.5 years developed colo-vesical fistula that required fistulectomy. Follow up colonoscopy showed severe ulcerations at the sigmoid and ascending colon. Anakinra, an IL1 receptor antagonist, was commenced with escalation of the dose up to $3 \mathrm{mg} / \mathrm{kg}$ daily, though clinical or laboratory responses were not documented. Importantly, the patient did not develop severe or atypical inflammation until the age of 3 years.

Patient 2 was also born to a consanguineous Muslim family (parents first degree cousins) and presented at the age of 1 month with recurrent fevers, non-bloody diarrhea, oral ulcers, poor weight gain, abdominal distention and arthritis. During infancy he developed recurrent perianal abscesses. At the age of 20 months his weight was $9.7 \mathrm{~kg}$ (-2 SD), length $81 \mathrm{~cm}$ (-1 SD), head circumference $46 \mathrm{~cm}(-1 \mathrm{SD})$. Physical exam was noted for abdominal distention and hepatosplenomegaly, along with a draining perianal abscess. Blood tests demonstrated anemia (hemoglobin $8.8 \mathrm{~g} / \mathrm{dL}$ ) and mildly elevated inflammatory markers (ESR 29 mm/hour). Blood cultures were positive for Providencia stuartii and Pseudomonas aeruginosa, which were treated with Piperacillin-Tazobactam for two weeks. Colonoscopy showed patchy colitis, predominantly at the right colon, while an upper endoscopy was unremarkable. Pathologic assessment showed patchy chronic active colitis. The patient temporarily responded to mesalamine, metronidazole and azathioprine, with a $3 \mathrm{~kg}$ weight gain over 10 months and normalization of inflammatory markers. However, he continued to suffer from recurrent febrile episodes, oral ulcers, diarrhea, arthritis and perianal abscesses.

\section{Basic immune studies}

Immunoglobulin levels, including $\lg M$, IgA and IgG were within normal limits for both patients (Supplemental Table 3). Moreover, immunoglobulin E was also normal for Patient 1. In addition, 
lymphocyte subset analysis was within normal limits for both patients ,beside slightly elevated CD8 ${ }^{+} \mathrm{T}$ cells (Supplemental Table 3). Finally, TREC levels for both patients were within normal limits, reflecting intact thymic function.

\section{Identification of RIPK1 mutation}

Following WES analysis, we identified 7,928 and 6,887 homozygous variants for Patients 1 and 2, respectively, that affect protein sequences. These numbers were reduced to 49 and 77 variants, respectively, after filtration for common variants (MAF $\geq 0.01$ ) in either our local in-house exomes database ( $n \sim 3500)$ or external databases such as 1000 Genomes Project (1 KG;

https://www.internationalgenome.org/1000-genomes-browsers) or dbSNP 135 database, the NHLBI Exome Sequencing Project (ESP) (http://evs.gs.washington.edu/EVS/) or gnomAD database (https://gnomad.broadinstitute.org/). A c.1934C>T missense mutation residing in Exon 11 was identified in Patient 1, and a c.580G>A RIPK1 missense mutation residing in Exon 4 was identified in Patient 2 (Figure 2). Both mutations were verified using Sanger sequencing, were designated as deleterious to protein function and were not found in gnomAD Database, dbSNP, 1000G and ESP6500, nor in our inhouse database.

\section{Abnormal Cytokine Production in RIPK1-deficient Patient}

To evaluate changes in the architecture and function of peripheral immune system in one of the RIPK1deficient patients, we performed mass cytometry (CyTOF) using a panel of 36 antibody markers, and compared the results to in comparison to both maternal and paternal blood samples, non-IBD subjects (controls) and CD patients, An unbiased clustering algorithm (Phenograph) was performed on multiple immune populations. To begin, we clustered on all immune cells $\left(\mathrm{CD} 45^{+}\right)$and were able to identify 28 unique populations (Figure $3 \mathrm{~A}-\mathrm{C}$ ). When comparing abundances of major immune subsets, we identified an increased abundance of T cells, particularly CD8 effector population, in the RIPK1-deficient patient, compared to controls, along with a decrease in all other cell types including monocytes, dendritic cells and B cells (Figure $3 \mathrm{C}-\mathrm{E}$ ). Interestingly, we also observed a reversal of the CD4 to CD8 ratio compared to all other samples (Figure 3F).

To gain further insight into the functional effects of RIPK1 deficiency, we examined cytokine production by PBMCs following stimulation with either LPS or PMA/lonomycin, using CyTOF, and clustering on all leukocytes (CD45 ${ }^{+}$cells, Supplemental Figure $1 \mathrm{~A}-\mathrm{C}$ ). We were able to identify 17 unique populations, again demonstrating a CD8 predominance in the patient (Supplemental Figure 1A-B). RIPK1-deficient patient's immune cells exhibited decreased IL-6 production in response to LPS, but not to PMA-I stimulation, across multiple cell types including $T$ cells, B cells and innate immune cells (Figure 4A). In addition, we observed an increase in IL-22 production across the majority of immune cells, particularly under the LPS stimulation condition (Figure 4B).

Focusing on the individual cell populations, monocytes and dendritic cell's ability to produce inflammatory cytokines was drastically reduced in the patient, consistent with documented reduced NFkB 
activity $^{17}$ (Figure 4C). On the other hand, patient's T cells produced higher amounts of IFNg than healthy controls upon PMA/I stimulation (Figure 4E). Interestingly, when analyzing immune populations with inhibitory potential, including myeloid derived suppressor cells (MDSC) and CD24 ${ }^{+} \mathrm{B}$ cells (containing the putative regulatory $\mathrm{B}$ cell populations), we demonstrate higher production of inflammatory cytokines (IL23, IL 1b, and IL-8), but also IL-10, compared with cells obtained from the parents (Figure 4C), suggesting a possible dysfunction of these cells.

\section{Discussion}

With the expanding use of next generation sequencing platforms in patients with unique IBD phenotypes, the list of monogenic disorders associated with intestinal inflammation is steadily growing, and many of them present with an increased susceptibility to severe and/or atypical infections. RIPK1 is a central regulator of apoptosis, inflammation, and necroptosis, and therefore, it is not surprising that patients that lack RIPK1-dependent signaling develop a severe multi-systemic disease with inflammatory phenotypes and infectious complications in some of them. To date, 14 patients with deleterious RIPK1 mutations have been reported in the literature ${ }^{9,10,18,19}$. All patients developed colitis, mostly in the first months of life, and in all but two patients perianal inflammation (abscesses or fistulas) was evident ${ }^{9,10}$. Chronic upper gastrointestinal tract inflammation was also reported ${ }^{18}$. Additional clinical features included susceptibility to severe viral and bacterial infections, arthritis, skin lesions, recurrent fever and oral ulcers. Differences in clinical phenotype may be attributed to the functional consequences of the RIPK1 mutation and whether it abrogates protein expression. This disorder should not be confused with a different autosomal dominant autoinflammatory disease caused by heterozygote mutations in RIPK1, leading to its abnormal cleavage by caspase- 8 , and manifesting as recurrent fever, lymphadenopathy and hepatosplenomegaly 20 .

One of the pathways that has been shown to be upregulated in RIPK1-deficient macrophages is activation of the inflammasome, an intracellular complex that secretes the pro-inflammatory cytokines IL$1 \mathrm{~b}$ and IL-18 in response to different stimuli, including lipopolysaccharide ${ }^{21}$. Impaired inflammasome activation has been reported in other monogenic disorders associated with intestinal inflammation, including Caspase-8 deficiency ${ }^{21}$, mutations in IL-10 receptor (IL10R) ${ }^{7}$, mevalonate kinase (MVK) deficiency ${ }^{22}$ and in gain-of-function mutations of $N L R C 4^{23}$. We have previously shown than anakinra, an IL1 receptor antagonist, was effective in suppressing intestinal inflammation in two patients with deleterious IL 1ORA mutations and history of severe infantile-onset IBD ${ }^{7}$, and, similarly, blocking IL-1 is effective in patients with MVK deficiency ${ }^{24}$. Over-activation of the inflammasome in patients with RIPK1 mutations may suggest that IL-1 blocking agents can be effective in suppressing the IBD phenotype, though additional pro-inflammatory cytokines (e.g. IL6) and pathways may also be dysregulated and contribute to the hyper-inflammatory immune response observed in this disorder. A trial of anakinra (IL-1 receptor antagonist) was performed in Patient 1 , but the dose provided was only $3 \mathrm{mg} / \mathrm{kg}$, much lower than the does we successfully used for the IL10R-deficient patients $(10 \mathrm{mg} / \mathrm{kg})^{7}$. 
Our CyTOF data of PBMCs from a single RIPK1-deficient patient highlighted alterations in both innate and adaptive immune cells. The patient displayed increased frequency of naïve $\mathrm{CD} 4^{+}$and $\mathrm{CD} 8^{+} \mathrm{T}$ cells, compared to controls and also to patients with active CD, similar to observations by $L i$ and colleagues 9 . In addition, we demonstrated decreased IL- 6 production by various innate and adaptive immune subsets. This finding is in-line with reports from Cuchet-Lourenco and colleagues who found that RIPK-deficient fibroblasts have reduced ERK and NfkB phosphorylation and a reduction in pro-inflammatory cytokines

such as IL-6, and that the TLR4 (LPS) signaling is affected more than TLR independent pathways ${ }^{10}$. The dysfunction of B cells and MDSC has been reported in IBD patients ${ }^{25,26}$; however, this is the first reporting of our knowledge in RIPK1 deficient patients.

The question whether hematopoietic stem cell transplantation (HSCT) can cure RIPK1 deficiency is unclear at this point. Cuchet-Lourenço and colleagues reported 3 siblings (One was sequenced and found to harbor a RIPK1 mutation and the others with the presumable same mutation) that underwent HSCT; two of them died within weeks of the procedure due to multi-organ failure or disseminated viral infections. The third patient underwent HSCT at the age of 30 months with resolution of IBD and arthritis (up to 5 years of follow-up), though is still on antibiotics due to chronic and probably irreversible lung disease ${ }^{10}$. Mice with a specific deletion of RIPK1 in epithelial cells develop spontaneous lethal intestinal inflammation due to enhanced apoptosis and necroptosis in epithelial cells ${ }^{27,28}$. Moreover, RIPK1deficient intestinal epithelial cells show altered cell death responses in response to TNFa stimulation ${ }^{9}$. Given RIPK1's roles in governing both immune and epithelial responses, clinicians should be cautious about the ability of HSCT to cure these patients, as it might ameliorate the immunodeficiency phenotype but not intestinal inflammation. This might be similar to Nuclear factor-kappa B essential modulator (NEMO) deficiency due to IKBKG mutations, in which HSCT eliminates the increased susceptibility to recurrent/atypical infections, but does not cure the IBD phenotype ${ }^{29}$.

In conclusion, mutations in RIPK1 are a new monogenic form of IBD that should be suspected in patients with very early-onset IBD with inflammatory and fistulizing features, along with immunodeficiency. Given RIPK1's involvement in regulating inflammasome function it is plausible that targeting IL-1 and/or IL-18 may be effective, to some extent, in suppressing intestinal inflammation. Nevertheless, murine and human data, as well as in vitro studies, suggest that RIPK1 is important for epithelial cell function, and therefore, therapies targeting immune cells may be only partially effective in these patients. Moreover, the ability of HSCT to cure this disorder is still unclear. Additional human studies and observations are required to determine the role of RIPK1 in epithelial cells in sustaining mucosal homeostasis.

\section{Declarations}

\section{Funding}

This study was funded by the Leona M. and Harry B. Helmsley Charitable Trust.

\section{Conflict of interest}


DSS declares speaker's fees from Abbvie and research grant from Takeda. SBS declares the following interests: Scientific advisory board participation for Pfizer, Pandion, Celgene, Lilly, Takeda, Cosmo Pharmaceuticals, Merck, Sonoma Biotherapeutics, and EcoR1. Grant support Pfizer, Amgen Takeda, and Novartis. Consulting for Amgen, Kyverna, Bristol Myers Squibb, Third Rock, 89bio, GentiBio and Apple Tree Life Sciences. All other authors do not have any other conflicts to disclose.

\section{Data availability}

The datasets generated during and/or analysed during the current study are available from the corresponding author on reasonable request.

\section{Authors' contribution}

MZ, MA, IA - data collection, manuscript editing

NK, BM, LW, AL, OB, LK, RZ - data analysis, manuscript editing

SBS - conceptualizing of study, data analysis, manuscript editing

DSS - conceptualizing of study, coordination of study, data analysis, manuscript writing

The study was approved by the IRB committee at Sheba Medical Center and informed consent was obtained from all individual participants included in the study or their parents.

\section{Ethical approval}

The study was approved by the IRB committee at Sheba Medical Center.

\section{Consent to participate}

Informed consent was obtained from all individual participants included in the study or their parents.

\section{Consent to publish}

The authors affirm that human research participants provided informed consent for publication of the images in Figure 1.

\section{References}

1. Chang, J. T. Pathophysiology of Inflammatory Bowel Diseases. N Engl J Med 383, 2652-2664, doi:10.1056/NEJMra2002697 (2020).

2. Graham, D. B. \& Xavier, R. J. Pathway paradigms revealed from the genetics of inflammatory bowel disease. Nature 578, 527-539, doi:10.1038/s41586-020-2025-2 (2020). 
3. Ouahed, J. et al. Very Early Onset Inflammatory Bowel Disease: A Clinical Approach With a Focus on the Role of Genetics and Underlying Immune Deficiencies. Inflamm Bowel Dis 26, 820-842, doi:10.1093/ibd/izz259 (2020).

4. de Mesquita, M. B. \& Shouval, D. S. Evaluation of very early-onset inflammatory bowel disease. Curr Opin Gastroentero/ 36, 464-469, doi:10.1097/MOG.0000000000000680 (2020).

5. Bolton, C. et al. An Integrated Taxonomy for Monogenic Inflammatory Bowel Disease. Gastroenterology, doi:10.1053/j.gastro.2021.11.014 (2021).

6. Nambu, R. et al. A Systematic Review of Monogenic Inflammatory Bowel Disease. Clin Gastroenterol Hepatol, doi:10.1016/j.cgh.2021.03.021 (2021).

7. Shouval, D. S. et al. Interleukin 1 beta Mediates Intestinal Inflammation in Mice and Patients With Interleukin 10 Receptor Deficiency. Gastroenterology 151, 1100-1104, doi:10.1053/j.gastro.2016.08.055 (2016).

8. Vardi, I. et al. Monogenic Inflammatory Bowel Disease: It's Never Too Late to Make a Diagnosis. Front Immuno/ 11, 1775, doi:10.3389/fimmu.2020.01775 (2020).

9. Li, Y. et al. Human RIPK1 deficiency causes combined immunodeficiency and inflammatory bowel diseases. Proc Natl Acad Sci U S A 116, 970-975, doi:10.1073/pnas.1813582116 (2019).

10. Cuchet-Lourenco, D. et al. Biallelic RIPK1 mutations in humans cause severe immunodeficiency, arthritis, and intestinal inflammation. Science 361, 810-813, doi:10.1126/science.aar2641 (2018).

11. Liu, Z. \& Chan, F. K. Regulatory mechanisms of RIPK1 in cell death and inflammation. Semin Cell Dev Biol, doi:10.1016/j.semcdb.2020.06.013 (2020).

12. Li, H. \& Durbin, R. Fast and accurate short read alignment with Burrows-Wheeler transform. Bioinformatics 25, 1754-1760, doi:10.1093/bioinformatics/btp324 (2009).

13. McKenna, A. et al. The Genome Analysis Toolkit: a MapReduce framework for analyzing nextgeneration DNA sequencing data. Genome Res 20, 1297-1303, doi:10.1101/gr.107524.110 (2010).

14. Li, M. X., Gui, H. S., Kwan, J. S., Bao, S. Y. \& Sham, P. C. A comprehensive framework for prioritizing variants in exome sequencing studies of Mendelian diseases. Nucleic Acids Res 40, e53, doi:10.1093/nar/gkr1257 (2012).

15. Werner, L. et al. Alterations in T and B Cell Receptor Repertoires Patterns in Patients With IL10 Signaling Defects and History of Infantile-Onset IBD. Front Immunol 11, 109, doi:10.3389/fimmu.2020.00109 (2020).

16. Konnikova, L. et al. High-dimensional immune phenotyping and transcriptional analyses reveal robust recovery of viable human immune and epithelial cells from frozen gastrointestinal tissue. Mucosal Immunol 11, 1684-1693, doi:10.1038/s41385-018-0047-y (2018).

17. Kondylis, V., Kumari, S., Vlantis, K. \& Pasparakis, M. The interplay of IKK, NF-kappaB and RIPK1 signaling in the regulation of cell death, tissue homeostasis and inflammation. Immunol Rev 277, 113-127, doi:10.1111/imr.12550 (2017). 
18. Uchiyama, Y. et al. Primary immunodeficiency with chronic enteropathy and developmental delay in a boy arising from a novel homozygous RIPK1 variant. J Hum Genet 64, 955-960, doi:10.1038/s10038019-0631-3 (2019).

19. Lin, L. et al. Clinical phenotype of a Chinese patient with RIPK1 deficiency due to novel mutation. Genes Dis 7, 122-127, doi:10.1016/j.gendis.2019.10.008 (2020).

20. Tao, P. et al. A dominant autoinflammatory disease caused by non-cleavable variants of RIPK1. Nature 577, 109-114, doi:10.1038/s41586-019-1830-y (2020).

21. He, Y., Hara, H. \& Nunez, G. Mechanism and Regulation of NLRP3 Inflammasome Activation. Trends Biochem Sci 41, 1012-1021, doi:10.1016/j.tibs.2016.09.002 (2016).

22. Akula, M. K. et al. Control of the innate immune response by the mevalonate pathway. Nat Immunol 17, 922-929, doi:10.1038/ni.3487 (2016).

23. Canna, S. W. et al. Life-threatening NLRC4-associated hyperinflammation successfully treated with IL-18 inhibition. J Allergy Clin Immuno/ 139, 1698-1701, doi:10.1016/j.jaci.2016.10.022 (2017).

24. Jeyaratnam, J. \& Frenkel, J. Management of Mevalonate Kinase Deficiency: A Pediatric Perspective. Front Immunol 11, 1150, doi:10.3389/fimmu.2020.01150 (2020).

25. Timmermans, W. M. et al. B-Cell Dysregulation in Crohn's Disease Is Partially Restored with Infliximab Therapy. PLoS One 11, e0160103, doi:10.1371/journal.pone.0160103 (2016).

26. Ostanin, D. V. \& Bhattacharya, D. Myeloid-derived suppressor cells in the inflammatory bowel diseases. Inflamm Bowel Dis 19, 2468-2477, doi:10.1097/MIB.0b013e3182902b11 (2013).

27. Dannappel, M. et al. RIPK1 maintains epithelial homeostasis by inhibiting apoptosis and necroptosis. Nature 513, 90-94, doi:10.1038/nature13608 (2014).

28. Takahashi, N. et al. RIPK1 ensures intestinal homeostasis by protecting the epithelium against apoptosis. Nature 513, 95-99, doi:10.1038/nature13706 (2014).

29. Miot, C. et al. Hematopoietic stem cell transplantation in 29 patients hemizygous for hypomorphic IKBKG/NEMO mutations. Blood 130, 1456-1467, doi:10.1182/blood-2017-03-771600 (2017).

\section{Figures}

\section{Figure 1}

Presenting features of the patients. (A) Perianal fistula extending to the base of the scrotum in Patient 1. (B) Hematoxylin and eosin stain of rectal biopsy demonstrating chronic active colitis.

\section{Figure 2}


Identification of RIPK1 mutations in index patients. Figure depicts chromatogram of RIPK1 gene sequencing at the site of designated mutations for (A) Patient 1 and (B) Patient 2.

\section{Figure 3}

Alterations in circulating leukocytes in a patient with RIPK1 deficiency. tSNE of Phenograph analysis of PBMCs as a (A) conglomerate of all samples with individual cellular population shown or as (B) density plots of the individual groups. (C) Heatmap of the markers expressed in each cluster on the left-hand-side, cluster abundance as a percent of all leukocytes in the middle and fold change of each cluster for each group shown as compared to the RIPK1 patient. (D-E) Clusters combined by the immune populations indicated. (F) CD4 to Cd8 ratio. Blue arrow-relative reduction and red arrow-relative increase in the RIPK1 patient compared to all other groups.

\section{Figure 4}

Changes in the cytokine profiles of PBMCs in a patient with RIPK1 deficiency. (A) IL-6 and (B) IL-22 expression in the various populations indicated obtained from phonograph analysis of stimulated PBMCS with either LPS or PMA+. (C) Cytokine production by the immune populations indicated. (D) IFNg production with stimulation by LPS or PMA/I in T cells.

\section{Supplementary Files}

This is a list of supplementary files associated with this preprint. Click to download.

- RIPK1Suppflies1002.pdf 